\title{
ON LATTICES OF RADICALS IN THE CLASS OF ALL FINITE GROUPS
}

\author{
JAN KREMPA $^{\circledR}$ and IZABELA AGATA MALINOWSKA
}

(Received 29 November 2011)

\begin{abstract}
Lattices of radicals have been extensively studied, for example in the class of associative rings, leading to some interesting results. In this paper we investigate the lattice $\mathbf{L}$ of all radicals in the class of all finite groups. We also consider some of its important sublattices. In particular, we prove that the lattice $\mathbf{L}$ is closed to being modular, the lattice $\mathbf{L}_{h}$ of all hereditary radicals is a Boolean algebra, and there exists a natural, useful projection of the lattice $\mathbf{L}$ onto $\mathbf{L}_{h}$.
\end{abstract}

2010 Mathematics subject classification: primary 20D30; secondary 06C99, 16N80.

Keywords and phrases: finite groups, radicals, hereditary radicals, simple groups, lattices of radicals, balanced lattices.

\section{Preliminaries}

All groups considered in this paper are finite. Let $G$ be a group. Then it is well known (see $[2,10]$ ) that every minimal subnormal subgroup of $G$ is a simple group, while every minimal normal subgroup of $G$ is a direct power of a simple group. Hence, every composition factor of $G$ is a simple group, while every chief factor of $G$ is a direct power of a simple group. By the celebrated Jordan-Hölder theorem, to any group $G$ a composition length $\lambda(G)$ is assigned.

In the sequel $\mathfrak{F}$ will denote the class of all finite groups. All classes of groups considered are subclasses of $\mathfrak{F}$, are closed under taking isomorphic images and contain the trivial group 1 . Our universal class $\mathfrak{F}$ contains, up to isomorphism, only $\boldsymbol{\aleph}_{0}$ objects, thus only $2^{\aleph_{0}}$ subclasses. Hence the subclasses form a set, say $2^{\mathfrak{r}}$, and it is a Boolean algebra under natural inclusion of classes, with distinguished elements (1) and $\mathfrak{F}$. Further every subset of $2^{\mathfrak{\mho}}$ will be considered as ordered by inclusion, often indicated as inequality.

If $\mathfrak{X}$ is a family of groups, then we use $(\mathfrak{X})$ to denote the smallest class of groups containing $\mathfrak{X}$. Hence every group from $(\mathfrak{X})$ is either isomorphic to a group from $\mathfrak{X}$, or is trivial.

(c) 2012 Australian Mathematical Publishing Association Inc. 0004-9727/2012 \$16.00 
We will use here some standard operators with values in classes of groups. Let $\mathfrak{X}$ be a family of groups. Then $Q \mathfrak{X}$ denotes the homomorphic closure of $\mathfrak{X}, \mathcal{H} \mathfrak{X}$ denotes the class of all groups isomorphic to subnormal subgroups of groups from $\mathfrak{X}$ and $\mathcal{E} \mathfrak{X}=\mathfrak{X} \mathfrak{X}$ denotes the class of all extensions of groups from $\mathfrak{X}$ by groups from $\mathfrak{X}$. By $\overline{\mathcal{E}} \mathfrak{X}$ we denote the smallest class containing $\mathfrak{X}$ and closed under extensions. We have $\overline{\mathcal{E}} \mathfrak{X}=\bigcup_{n=1}^{\infty} \mathcal{E}^{n} \mathfrak{X}$. This means that $G \in \overline{\mathcal{E}} \mathfrak{X}$ if and only if $G$ has a subnormal series with all factors in $\mathfrak{X}$.

In line with terminology used in radical theory, let us agree that a class $\mathfrak{X}$ is hereditary if $\mathfrak{X}=\mathcal{H} \mathfrak{X}$. An easy consequence of the isomorphism theorems and finiteness of our groups is the following lemma.

LEMMA 1.1. Let $\mathfrak{C}$ be a class of groups.

(i) If $\mathbb{C}$ is hereditary, then $\mathcal{E} \mathbb{C}, \overline{\mathcal{E}} \mathfrak{C}$ and $Q \mathbb{C}$ are also hereditary.

(ii) If $\mathbb{C}$ is closed under homomorphisms, then $\mathcal{E} \mathfrak{C}$ and $\overline{\mathcal{E}} \mathfrak{C}$ are also closed under homomorphisms.

(iii) If $1 \neq \mathbb{C}$ is either closed under homomorphisms or is hereditary, then $\mathbb{C}$ contains a simple group.

All radicals in this paper are understood in the sense of Kurosh and Amitsur. They will be considered in the class $\mathfrak{F}$. One can find the basics on such radicals in, for example, [7]. One can also adopt results on radicals from some other universal classes [3, 4, 16], but keeping in mind Lemma 1.1(iii) and the Jordan-Hölder Theorem. However, let us recall some basic results, for completeness.

A class $\mathfrak{R}$ of groups is a radical class or simply a radical if it has the following properties.

(I) $\mathfrak{R}=Q \Re$.

(II) For every group $G$, the join $\mathfrak{R}(G)=\langle H \triangleleft G \mid H \in \mathfrak{R}\rangle$ is in $\Re$.

(III) $\mathfrak{R}(G / \mathfrak{R}(G))=1$ for every group $G$.

The subgroup $\mathfrak{R}(G)$ is called the $\mathfrak{R}$-radical (a radical) of $G$ and $G / \mathfrak{R}(G)$ is called an $\Re$-semisimple (semisimple) image of $G$. In this way to any radical class $\Re$ there corresponds the semisimple class

$$
\mathcal{S} \Re=\{G \mid \mathfrak{R}(G)=1\} .
$$

As an immediate consequence we obtain the following well-known property. Let $\mathfrak{R}$ be a radical class. Then $\mathfrak{R}(G)$ is a characteristic subgroup of $G$ for any group $G$. Thus, if $N \triangleleft G$ then $\mathfrak{R}(N) \triangleleft G$ and $\mathfrak{R}(N) \leqslant \mathfrak{R}(G)$. In particular, the class $\mathcal{S} \Re$ is hereditary.

EXAMPLE 1.2. Let $\mathfrak{C}$ be a hereditary class of groups and let $\mathcal{U} \mathfrak{C}$ be the class of all groups $G$ having no homomorphisms onto a nontrivial $\mathfrak{C}$-group. It follows immediately from the definition that the class $\mathcal{U} \mathfrak{C}$ is a radical class. This class is called the upper radical determined by $\mathfrak{C}$, because it is the largest among radicals $\mathfrak{R}$ with $\mathfrak{C} \subseteq \mathcal{S} \Re$.

Note that $\mathcal{U} \mathfrak{C}$-groups are groups equal to their $\mathfrak{C}$-residuals. However, for a group $G$ the radical $\mathcal{U} \mathfrak{C}(G)$ need not be equal to the $\mathfrak{C}$-residual of $G$. 
Our groups are finite. Hence (see [7]) we have the following theorem.

THEOREM 1.3. Let $\mathfrak{C}$ be a class of groups.

(i) $\mathfrak{C}$ is a radical class if and only if $\mathfrak{C}=Q \mathbb{C}=\mathcal{E} \mathfrak{C}$.

(ii) $\mathfrak{C}$ is an $\mathfrak{R}$-semisimple class for a radical class $\mathfrak{R}$ if and only if $\mathfrak{C}=\mathcal{H} \mathfrak{C}=\mathcal{E} \mathfrak{C}$.

Further, for any family of groups $\mathfrak{C}$, we will consider the following classes: the lower radical class $\mathcal{L} \mathfrak{C}$ containing $\mathfrak{C}$, the lower hereditary radical class $\mathcal{L}_{h} \mathfrak{C}$ containing $\mathfrak{C}$ and the lower semisimple class, say $\mathcal{M} \mathfrak{C}$, containing $\mathfrak{C}$. Here, according to radical tradition, the lower means the smallest.

With the help of intersection of classes and Theorem 1.3 we immediately obtain that, for any family $\mathfrak{C}$, the classes $\mathcal{L} \mathfrak{C}, L_{h} \mathfrak{C}$ and $\mathcal{M C}$ exist. However, more useful is the following effective description of these classes.

THEOREM 1.4. Let $\mathfrak{C}$ be a family of groups. Then:

$$
\mathcal{L} \mathfrak{C}=\overline{\mathcal{E}} Q \mathfrak{C}, \quad \mathcal{L}_{h} \mathfrak{C}=\overline{\mathcal{E}} Q \mathcal{H} \mathfrak{C}, \quad \text { and } \quad \mathcal{M} \mathfrak{C}=\overline{\mathcal{E}} \mathcal{H} \mathfrak{C} .
$$

\section{Some connections with simple groups}

In the sequel $\subseteq$ will denote the family of all simple groups. If $\mathfrak{I} \subseteq \subseteq$ is any family of simple groups then $(\mathfrak{I})$ will be called a class of simple groups.

As a slight modification of results from [7] we have the following characterisation of hereditary radical classes, semisimple classes closed under homomorphisms and radical semisimple classes.

THeOREM 2.1. Let $\mathfrak{R}$ be a class of groups. Then the following conditions are equivalent:

(i) $\Re$ is a hereditary radical;

(ii) $\mathfrak{R}$ is the lower radical of a hereditary class;

(iii) $\mathfrak{R}$ is the lower radical of a class of simple groups;

(iv) $\mathfrak{R}=\overline{\mathcal{E}} \mathfrak{I}$ where $\mathfrak{I}$ is the class of all simple $\mathfrak{R}$-groups;

(v) $\mathfrak{R}$ is a radical and semisimple class;

(vi) $\mathfrak{R}=\mathcal{H} \Re=Q \Re=\mathcal{E} \Re$;

(vii) $\Re$ is a semisimple class closed under homomorphisms.

In connection with the above theorem let us agree that a radical $\Re$ is cohereditary if its semisimple class $\mathcal{S}(\mathfrak{R})$ is closed under homomorphisms. With the help of Theorem 2.1 and Lemma 1.1 cohereditary radicals can be characterised in the following way.

THEOREM 2.2. Let $\mathfrak{R}$ be a class of groups. Then $\Re$ is a cohereditary radical if and only if $\mathcal{S}(\mathfrak{R})$ is a hereditary radical class. In particular, upper radicals for classes of simple groups are cohereditary. 
The classes (1) and $\mathfrak{F}$ are trivial radicals. There are also other natural, useful examples of radicals.

ExAmple 2.3. The class $\mathfrak{D}$ of all solvable groups is a hereditary radical class. Hence it is also a semisimple class closed under taking quotient images. Further, $\mathfrak{D}$ is the lower radical of the class of all simple abelian groups.

On the other hand, the class $\mathfrak{N}$ of all nilpotent groups is neither radical nor semisimple, because it is not closed under extensions.

EXAmple 2.4. Let $\pi$ be a set of prime numbers. Then the class $\mathfrak{F}_{\pi}$ of all $\pi$-groups is a hereditary radical class. Hence it is also a semisimple class closed under taking quotient images. Further, $\mathfrak{F}_{\pi}$ is the lower radical of all simple $\pi$-groups.

ExAmple 2.5. The class $\mathfrak{P}$ of all perfect groups (groups $G$ with $G=G^{\prime}$ ) is a radical class. One can check that $\mathfrak{P}=\mathcal{U}(\mathfrak{D})$, so that it is the upper radical of all simple abelian groups. The class $\mathfrak{P}$ is not a hereditary radical class, but is cohereditary.

Further, the (regular) wreath product (see $[2,12])$ will be a helpful construction. This construction is defined as follows. Let $A, B$ be groups and let $A^{B}=F$ be the group of all functions from $B$ to $A$ with natural pointwise multiplication. Then $F$ is the direct product of $|B|$ isomorphic copies of $A$. Now let $b \in B$. If $f \in F$, define $\sigma_{b}(f)=f^{b}$ by

$$
f^{b}(x)=f\left(x b^{-1}\right) \text { for all } x \in B .
$$

The set of automorphisms $\left\{\sigma_{b}: b \in B\right\}$ is a group isomorphic to $B$ in a natural way. We shall identify these groups. The wreath product $W=A<B$ of $A$ by $B$ is the semidirect product of $F$ by this group of automorphisms; that is, $W=B F$ with the relations

$$
b f c g=b c f^{c} g \text { for all } b, c \in B \text { and } f, g \in F .
$$

One can check that if $1 \neq N \triangleleft W$, then $N \cap F \neq 1$. As a consequence one can obtain that if $A$ is simple and either is nonabelian, or $|A|$ and $|B|$ are coprime, then $F$ is a minimal normal subgroup of $A$ < $B$.

As a consequence of the above result and properties of radicals, we obtain the following useful observation.

Lemma 2.6. Let $A, B$ be groups and $\Re$ be a radical class.

(i) If $A, B \in \mathfrak{R}$, then $A$ 乙 $B \in \mathfrak{R}$.

(ii) If $A, B \in \mathcal{S} \Re$, then $A$ 乙 $B \in \mathcal{S} \Re$.

(iii) If $A \in \mathfrak{R}$ and $B \in \mathcal{S} \Re$, then $\mathfrak{R}(A<B)$ is the base group of $A$ < $B$.

(iv) If $\Re$ is hereditary and $1 \neq A \in \mathcal{S} \Re$, then $A$ < $B \in \mathcal{S} \Re$.

(v) Let $A \in \mathcal{S} \Re$ be simple and $B \in \mathfrak{R}$. If either $A$ is nonabelian, or $|A|$ and $|B|$ are coprime, then either $A$ ᄂ $B \in \mathcal{S} \Re$ or $A$ ᄂ $B \in \mathfrak{R}$.

Proof. Claims (i)-(iv) follow easily from the definition of radicals. Now let $A$ and $B$ satisfy one of the assumptions of (v), $G=A \succ B$ and $F$ be the base group of $G$. Using the assumption, we already know that $F$ is a minimal normal subgroup of $G$. 
If $\mathfrak{R}(G) \neq 1$ then $\mathfrak{R}(G) \cap F \neq 1$. By minimality of $F$ in $G$, we know that $F \subseteq \Re(G)$. Thus $G / \mathfrak{R}(G)$ is a homomorphic image of an $\mathfrak{R}$-group $G / F \simeq B$. This means that $\Re(G)=G$.

As a consequence we obtain the following lemma.

LEMMA 2.7. Let $\mathfrak{I}$ be a nontrivial (or proper) subclass of the class $\mathfrak{\subseteq}$. Then the radicals $\mathcal{L} \mathfrak{I}$ and $\mathcal{U}(\mathfrak{S} \backslash \mathfrak{I})$ are different.

Proof. Let $\mathcal{L}(\mathfrak{I})=\mathfrak{L}$ and $\mathcal{U}(\mathfrak{S} \backslash \mathfrak{I})=\mathfrak{U}$. Clearly $\mathfrak{L} \leq \mathfrak{U}$. By assumption on $\mathfrak{I}$ there are simple groups $B \in \mathfrak{I}$, and $A \in \mathfrak{S} \backslash \mathfrak{I}$. Let $G=A$ 乙 $B$. By Lemma 2.6(iv) we have $\mathfrak{L}(G)=1$, because $\mathfrak{L}$ is hereditary and $A \notin \mathfrak{L}$.

On the other hand, one can check that $\mathfrak{U}(G) \neq 1$, because $\mathfrak{U}$ is cohereditary by Theorem 2.2, and $G$ has a homomorphic image isomorphic to $B \in \mathfrak{I}$. Hence, $\mathfrak{Q} \neq \mathfrak{U}$.

THeORem 2.8. Let $\mathfrak{R}$ be a hereditary and cohereditary radical. Then $\mathfrak{R}$ is trivial.

Proof. Assume that $\mathfrak{R}$ is hereditary and cohereditary, but is nontrivial. By Theorem 2.1 there is a nontrivial class $\mathfrak{I}$ of simple groups such that $\mathfrak{R}=\mathcal{L} \mathfrak{I}$. On the other hand, by the definition and Theorem $2.2, \mathfrak{R}=\mathcal{U} \mathfrak{I}^{\prime}$, for a nontrivial class $\mathfrak{I}^{\prime}$ of simple groups, because $\mathfrak{R}$ is cohereditary. One can check that $\mathfrak{I}^{\prime}=\mathfrak{S} \backslash \mathfrak{I}$. Hence, $\mathcal{L} \mathfrak{I}=\mathcal{U}(\mathfrak{S} \backslash \mathfrak{I})$, contradicting the preceding lemma.

\section{The lattice of all radicals}

In several universal classes radicals are not sets. However they are considered as lattices. Such lattices have been extensively studied, for example, in the class of all associative rings and in its subclasses (see $[4,13,15,17]$ and references therein). This approach has been fruitful for both ring theory and lattice theory.

In this section we will deal with the class, and in fact the set, $\mathbf{L}$ of all radical classes in $\mathfrak{F}$, ordered by inclusion. For the notation and terminology on lattices used below we refer the reader to [5]. As expected, we have the following theorem.

THeorem 3.1. The set $\mathbf{L}$ forms a complete lattice, and for any collection $\left\{\Re_{i} \mid i \in I\right\}$ of radicals,

$$
\inf _{i \in I} \Re_{i}=\bigcap_{i \in I} \Re_{i} \quad \text { and } \quad \sup _{i \in I} \Re_{i}=\mathcal{L}\left(\bigcup_{i \in I} \mathfrak{R}_{i}\right)=\overline{\mathcal{E}}\left(\bigcup_{i \in I} \mathfrak{R}_{i}\right) .
$$

If $\left\{\Re_{i}\right\}$ is a chain then $\sup _{i \in I} \Re_{\mathrm{i}}=\bigcup_{i \in I} \Re_{i}$. The lattice $\mathbf{L}$ is lower and upper continuous, but is not a sublattice of $2 \widetilde{\mho}$.

Proof. The first claim is obvious from the definition. The formula for upper bounds of chains follows, because every group has, up to isomorphism, only finitely many sections. The other claims are consequences of general properties of constructions for lower radicals. 
The set $\mathbf{M}$ of all semisimple classes in $\mathfrak{F}$ is also a complete lattice under inclusion, with operations given for any family $\left\{X_{i} \mid i \in I\right\}$ of semisimple classes by the formulas

$$
\inf _{i \in I} \mathfrak{X}_{i}=\bigcap_{i \in I} \mathfrak{X}_{i} \quad \text { and } \quad \sup _{i \in I} \mathfrak{X}_{i}=\mathcal{M}\left(\bigcup_{i \in I} \mathfrak{X}_{i}\right)=\overline{\mathcal{E}}\left(\bigcup_{i \in I} \mathfrak{X}_{i}\right) \text {. }
$$

If $\left\{\mathfrak{X}_{i}\right\}$ is a chain then, as in the preceding theorem, $\sup _{i \in I} \mathfrak{X}_{i}=\bigcup_{i \in I} \mathfrak{X}_{i}$.

By formula (1.1), Example 1.2 and Theorem 1.3 there is a Galois correspondence between radical and semisimple classes.

THEOREM 3.2. The operator $\mathcal{S}$ defines a natural dual isomorphism between the lattice $\mathbf{L}$ and the lattice $\mathbf{M}$. Moreover, $\mathcal{S}^{-1}=\mathcal{U}$.

This theorem allows us to concentrate mainly on radical classes (as in [15]), and we will also do so here.

TheOrem 3.3. For the lattice $\mathbf{L}$ the following statements hold.

(1) Every atom in $\mathbf{L}$ is the lower radical determined by a simple group and every lower radical determined by a simple group is an atom in $\mathbf{L}$. Hence $\mathbf{L}$ is atomic.

(2) Every coatom in $\mathbf{L}$ is the upper radical determined by a simple group and every upper radical determined by a simple group is a coatom in $\mathbf{L}$. Hence $\mathbf{L}$ is coatomic.

(3) $\mathbf{L}$ is not atomistic.

(4) $\mathbf{L}$ is not coatomistic.

(5) Hereditary radicals and cohereditary radicals are distributive elements in $\mathbf{L}$.

Proof. (1) and (2) are easy consequences of Lemma 1.1(iii) and Theorem 1.4.

(3) By (1) and Theorem 2.1, every atom in $\mathbf{L}$ is hereditary. Thus the join of any family of atoms is hereditary too. On the other hand, there exist proper nonhereditary radicals by Theorem 2.8 and Lemma 2.7 . Hence $\mathbf{L}$ is not atomistic.

(4) By (2) and Theorem 2.2, every coatom in $\mathbf{L}$ is cohereditary. Thus the intersection of any family of coatoms in $\mathbf{L}$ is cohereditary. On the other hand, with the help of Theorem 2.8 and Lemma 2.7, there are hereditary radicals which are not cohereditary. Hence $\mathbf{L}$ is not coatomistic.

(5) Let $\mathfrak{R}$ be a hereditary radical and $\mathfrak{X}, \mathfrak{Y}$ be arbitrary radicals. By induction on the composition length $\lambda(G)$ of a group $G$, one can show that if $G \in(\mathfrak{R} \wedge(\mathfrak{X} \vee \mathfrak{Y}))$ then $G \in((\mathfrak{R} \wedge \mathfrak{X}) \vee(\Re \wedge \mathfrak{Y}))$. This gives the crucial inclusion of classes for the case of hereditary $\mathfrak{R}$.

The case where $\mathfrak{R}$ is cohereditary can be proved by analogous considerations on the level of semisimple classes and then translated to $\mathbf{L}$ by the Galois correspondence (see Theorem 3.2).

In the sequel let $\mathbf{B}=2^{\subseteq}$ denote the Boolean algebra of all subclasses of our class $\subseteq$ of simple groups. 
Theorem 3.4. The lattice $\mathbf{L}$ is complemented. More precisely, if $\Re$ is a radical class then a radical class $\mathfrak{C}$ is its complement in $\mathbf{L}$ if and only if $\mathfrak{C} \cap \mathfrak{S}$ is a complement of $\Re \cap \subseteq$ in $\mathbf{B}$. Thus every proper radical is not a modular element in $\mathbf{L}$. In particular, $\mathbf{L}$ is not a modular lattice.

Proof. Let $\mathfrak{R}$ be a radical and let $\mathfrak{I}=\mathfrak{R} \cap \mathfrak{S}$ be the class of all simple $\mathfrak{R}$-groups. Also let $\mathfrak{C}$ be a radical such that $\mathfrak{S} \backslash \mathfrak{I}$ is the class of all simple $\mathfrak{C}$-groups, for example $\mathfrak{C}=\mathcal{L}(\mathfrak{S} \backslash \mathfrak{I})$. As a consequence of Lemma 1.1(iii) and Theorem 3.3,

$$
\Re \wedge \mathfrak{C}=(1) \text { and } \mathfrak{R} \vee \mathfrak{C}=\mathfrak{F}
$$

Hence, $\mathfrak{C}$ is a complement of $\Re$ in $\mathbf{L}$.

Similar arguments can be applied in the proof that complements should have the desired form.

Now let $\mathfrak{M}$ be a nontrivial radical, $\mathfrak{I}=\mathfrak{M} \cap \mathfrak{S}, \mathfrak{U}$ be the upper radical determined by $\mathfrak{I}$ and $\mathfrak{L}$ be the lower radical determined by $\mathfrak{S} \backslash \mathfrak{I}$. Then, by the first part of the proof, $\mathfrak{L} \leq \mathfrak{U}$, both radicals are complements of $\mathfrak{M}$ in $\mathbf{L}$ and are different, by Lemma 2.7. From these properties,

$$
\mathfrak{L} \vee(\mathfrak{M} \wedge \mathfrak{U})=\mathfrak{L} \quad \text { while }(\mathfrak{L} \vee \mathfrak{M}) \wedge \mathfrak{U}=\mathfrak{U} \neq \mathfrak{L}
$$

Hence, $\mathfrak{M}$ is not a modular element of $\mathbf{L}$ and $\mathbf{L}$ is not a modular lattice.

From [14] and references therein, we know that several families of formations of finite groups are modular lattices. We will now show that the lattice $\mathbf{L}$ satisfies a weaker condition.

A lattice $\mathbf{L}$ is called balanced if, for all $x, y, z \in \mathbf{L}$,

$$
x \wedge y \wedge z=x \wedge y=(x \vee y) \wedge z=0 \Rightarrow(y \vee z) \wedge x=0
$$

$L$ is strongly balanced if each of its intervals is a balanced lattice. It is easy to check that modular lattices are strongly balanced. Balanced and strongly balanced lattices are interesting in their own right, as a generalisation of modular lattices. They are also basic, if one is going to apply the uniform dimension to lattices of subgroups (see [1, 8, 11]), or to other not necessarily modular lattices [9]. For example, this notion is applicable to sublattices of our lattice $\mathbf{L}$ and to their duals, because of the following result.

Theorem 3.5. The lattice $\mathbf{L}$ and its dual are strongly balanced.

Proof. Let $\mathfrak{X}, \mathfrak{Y}$ and $\mathfrak{Z}$ be radicals, and put $\mathfrak{C}=\mathfrak{X} \wedge \mathfrak{Y} \wedge \mathfrak{3}$. Assume that

$$
\mathfrak{C}=\mathfrak{X} \wedge \mathfrak{Y}=(\mathfrak{X} \vee \mathfrak{Y}) \wedge 3 .
$$

From these equalities one can obtain, even at lattice level, that

$$
\mathfrak{C}=\mathfrak{X} \wedge 3=\mathfrak{Y} \wedge \mathfrak{3}
$$


Now let $G \in(\mathfrak{Y} \vee \mathfrak{3}) \wedge \mathfrak{X}$. By induction on $\lambda(G)$ we will show that $G \in \mathfrak{C}$. Certainly $G \in \mathfrak{X}$. If $G=1$ then obviously $G \in \mathfrak{C}$. Let $G \neq 1$. Then by Theorems 1.4 and 3.1, either $\mathfrak{Y}(G)$ or $\mathfrak{3}(G) \neq 1$. For example, let $H=\mathfrak{Y}(G) \neq 1$. Then $\lambda(G / H)<\lambda(G)$ so, by the induction assumption, $G / H \in \mathfrak{C}$. Hence $G / H \in \mathfrak{Y}$ and $G \in \mathfrak{Y}$. Therefore $G \in \mathfrak{X} \wedge \mathfrak{Y}=\mathfrak{C}$. The case $3(G) \neq 1$ can be considered in an analogous way. Hence in both cases $G \in \mathfrak{C}$. This, by definition, means that $\mathbf{L}$ is strongly balanced.

Working with semisimple classes one can prove in an analogous way that $\mathbf{M}$, hence also the dual of $\mathbf{L}$, is a strongly balanced lattice.

\section{Some sublattices}

We will now discuss two subsets of $\mathbf{L}$ : the set $\mathbf{L}_{h}$ of all hereditary radicals, and the set $\mathbf{L}_{c h}$ of all cohereditary radicals, with order given by inclusion. Simple considerations and Theorems 1.4, 2.1 and 2.2 together yield the following theorem.

THeOREM 4.1. The sets $\mathbf{L}_{h}$ and $\mathbf{L}_{c h}$ are complete sublattices of $\mathbf{L}$.

Now let us focus on simple groups. As earlier, let $\mathbf{B}$ be the Boolean algebra of all classes of simple groups. For a subclass $\mathfrak{I} \subseteq \subseteq$ let $\mathcal{U}^{\prime} \mathfrak{I}=\mathcal{U}(\mathfrak{S} \backslash \mathfrak{I})$. The following theorem is a consequence of Theorems 2.1 and 2.2.

TheOREM 4.2. The operators $\mathcal{L}$ and $\mathcal{U}^{\prime}$ restricted to $\mathbf{B}$ give lattice isomorphisms of $\mathbf{B}$ onto $\mathbf{L}_{h}$, and $\mathbf{L}_{c h}$, respectively. Hence, $\mathbf{L}_{h}$ and $\mathbf{L}_{c h}$ are complete Boolean algebras and are isomorphic in a natural way.

Corollary 4.3. Intervals in $\mathbf{L}_{h}$ and in $\mathbf{L}_{c h}$ are atomistic, coatomistic, and complete Boolean algebras.

Let $\Re$ be a radical. Denote by $\tau(\Re)$ the largest hereditary radical contained in $\Re$ and by $v(\Re)$ the smallest cohereditary radical containing $\Re$. The existence of $\tau$ and $v$ is a consequence of Theorem 4.2. Using also some earlier results one can obtain the following theorems.

THEOREM 4.4. The formula $\Re \rightarrow \tau(\Re)$ gives a projection of the complete lattice $\mathbf{L}$ onto $\mathbf{L}_{h}$. Under this projection, for every $\Re_{1}, \Re_{2} \in \mathbf{L}, \tau\left(\Re_{1}\right)=\tau\left(\Re_{2}\right)$ if and only if $\mathfrak{R}_{1} \cap \mathfrak{S}=\mathfrak{R}_{2} \cap \mathfrak{S}$.

THEOREM 4.5. The formula $\mathfrak{R} \rightarrow v(\Re)$ gives a projection of the complete lattice $\mathbf{L}$ onto $\mathbf{L}_{c h}$. Under this projection, for every $\Re_{1}, \Re_{2} \in \mathbf{L}, v\left(\Re_{1}\right)=v\left(\Re_{2}\right)$ if and only if $\Re_{1} \cap \mathfrak{S}=$ $\Re_{2} \cap \mathfrak{S}$. In particular, $\tau$ and $v$ define the same congruence on $\mathbf{L}$ and, for every radical $\mathfrak{R}, \tau(\mathfrak{R}) \leq \Re \leq v \Re$. Moreover, $[\tau(\mathfrak{R}), v(\Re)] \cap \mathbf{L}_{\mathbf{h}}=\tau(\mathfrak{R})$ and $[\tau(\mathfrak{R}), v(\Re)] \cap$ $\mathbf{L}_{\mathbf{c h}}=v(\Re)$.

From the above results, we know that with any radical $\mathfrak{R}$ we have associated a 'small' interval $[\tau(R), v(\Re)] \ni \Re$ and this interval is uniquely determined by a subclass $\mathfrak{I} \subseteq \mathfrak{S}$. Due to Lemma 2.7 and Theorem 4.2, we know that for every proper radical 
this subclass is proper in $\subseteq$ and the associated interval contains at least two elements. We will show that, in fact, this interval is infinite.

THeOREM 4.6. If $\mathfrak{I} \subset \subseteq \subseteq$ is a nontrivial class of simple groups then the interval $\left[\mathcal{L} \mathfrak{I}, \mathcal{U}^{\prime} \mathfrak{T}\right]$ has infinite height and infinite width.

Proof. Let $\mathcal{L} \mathfrak{I}=\mathfrak{L}$ and $\mathcal{U}^{\prime} \mathfrak{I}=\mathfrak{U}$.

Case 1. $\mathfrak{S} \backslash \mathfrak{I}$ contains an infinite sequence of nonabelian groups, say $A_{1}, A_{2}, \ldots$ Let $B \in \mathfrak{I}$ be any simple group. For every $i \geq 1$, let $G_{i}=A_{i} \prec B, \mathfrak{L}_{i}=\mathcal{L}\left(\mathfrak{I} \cup G_{i}\right)$ and $\mathfrak{R}_{i}=\mathcal{L}\left(\mathfrak{I} \cup A_{i}\right)$. Then $\mathfrak{L}<\mathfrak{Q}_{i}<\mathfrak{U}$, because $\mathfrak{L}$ is hereditary and $\mathfrak{U}$ is cohereditary. Also $\mathfrak{L}_{i} \leq \mathfrak{R}_{i}$, for every $i \geq 1$. Radicals $\mathfrak{R}_{i}$ belong to $\mathbf{L}_{h}$; hence, by Theorem 3.3(5),

$$
\mathfrak{L} \leq\left(\bigvee_{j=1}^{i} \mathfrak{L}_{j}\right) \wedge \mathfrak{L}_{i+1} \leq\left(\bigvee_{j=1}^{i} \mathfrak{R}_{j}\right) \wedge \mathfrak{R}_{i+1}=\mathfrak{L}
$$

From the above formula one can easily obtain the infinite width and infinite height of our interval.

Case 2. $\mathfrak{S} \backslash \mathfrak{I}$ contains an infinite sequence of abelian groups. In this case, after choosing $B \in \mathfrak{I}$ we are able to take different, simple abelian groups $A_{1}, A_{2}, \ldots$ with $A_{i}$ and $B$ having coprime orders for every $i \geq 1$. Using arguments as above, we also obtain the desired conclusion.

Case 3. Let $A \in \mathfrak{S} \backslash \mathfrak{I}$ and let us have an infinite sequence of groups $B_{1}, B_{2}, \ldots \in \mathfrak{I}$ with orders coprime to $|A|$. For every $i \geq 1$, let $G_{i}=A \curlywedge B_{i}, \mathfrak{R}_{i}=\mathcal{L}\left(\mathfrak{I} \cup G_{i}\right)$ and $\mathfrak{R}_{i}=$ $\bigvee_{j=1}^{i} \mathfrak{L}_{j}$. Then, for every $i \geq 1$,

$$
\mathfrak{L}<\mathfrak{L}_{i}<\mathfrak{R}_{i}<\mathfrak{U} \text { and } \mathfrak{L}_{i} \neq \mathcal{L} .
$$

Assume that, for some $i \geq 1, \mathfrak{Q}_{i+1}=\mathfrak{R}_{i}$. Then, in particular, $G_{i+1}$ contains a subnormal subgroup either from the class $\mathfrak{I}$ or from the set $\left\{G_{1}, \ldots G_{i}\right\}$. The first case is impossible, by our assumption on orders of $A$ and $B_{j}$. The second is also impossible, by comparing composition factors. Hence $\mathfrak{L}_{i+1} \neq \mathfrak{R}_{i}$.

From the above consideration one can easily obtain that radicals $\mathfrak{L}_{i}$ form an infinite antichain, while radicals $\mathfrak{R}_{i}$ form an infinite chain in our interval [ $\left.\mathfrak{L}, \mathfrak{U}\right]$.

Now it is easy to see that the three cases considered above give the proof of the theorem.

Another natural 'small' interval related to a subclass $\mathfrak{I}$ of simple groups could be of the form $[\mathcal{L} \mathfrak{I}, \mathcal{L}(\mathfrak{I} \cup A)]$, where $A$ is a simple group outside of $\mathfrak{I}$. In the lattice $\mathbf{L}_{h}$ this interval has only two elements. However, in many cases we can show that this interval is infinite in $\mathbf{L}$.

ExAmple 4.7. Let $\mathfrak{I} \subset \mathfrak{S}$ be a nontrivial class of simple groups, $A \in \mathfrak{S} \backslash \mathfrak{I}$ be nonabelian and $B \in \mathfrak{I}$. Put $G_{1}=A \curlywedge B$, and let $G_{n+1}=A$ 々 $G_{n}$ for $n \geq 1$. Let us consider radicals $\mathfrak{L}=\mathfrak{L}_{0}=\mathcal{L} \mathfrak{I}$ and as $\mathfrak{L}_{n}$ let us take the lower radical determined by $\mathfrak{L}$ and $G_{n}$ 
for $n \geq 1$. Let us also take $\mathfrak{B}=\mathcal{L}(\mathfrak{I} \cup A)$. By definition $G_{n}$ is a homomorphic image of $G_{n+1}$. Hence we have inequalities

$$
\mathfrak{L}=\mathfrak{Q}_{0} \leq \mathfrak{L}_{1} \leq \mathfrak{Q}_{2} \leq \cdots \leq \mathfrak{B}
$$

Using Lemma 2.6(v) and arguments as in the proof of Lemma 2.7, we can in fact say that $\mathfrak{L}_{0} \neq \mathfrak{L}_{1}$, because $G_{1} \notin \mathfrak{L}_{0}$ and $A$ is nonabelian.

Assume that $G_{n+1} \in \mathfrak{L}_{n}$ for some $n>1$ and that $n$ is minimal with this property. By Lemma 2.6(v), $G_{n+1}$ is $\mathfrak{Q}$-semisimple. Thus, by definition, $G_{n+1}$ contains a subnormal subgroup $H$ isomorphic to a homomorphic image of $G_{n}$, since $G_{n+1}$ is not an $\mathfrak{Q}$-group. Hence $H$ contains $B$ as a composition factor. On the other hand, the only subnormal subgroup of $G_{n+1}$ with $B$ as a composition factor has to be isomorphic to $G_{n+1}$, which is impossible. In this way we have proved that in the sequence (4.1) all inequalities are strict and our interval $[\mathfrak{L}, \mathfrak{B}]$ has infinite height.

Now, our groups $G_{n}$ and radicals $\mathfrak{R}_{n}$ depend also on $B \in \mathfrak{I}$. Hence, we can denote them by $\mathfrak{R}_{B, n}$ instead of $\mathfrak{L}_{n}$.

Under this notation one can prove that if $B \nsucc B^{\prime}$, with $B, B^{\prime} \in \mathfrak{I}$, then the radicals defined by these groups with the help of the same $A \in \mathfrak{S} \backslash \mathfrak{I}$ are distinct. The same holds for sequences based on $B^{\prime}$ and $A$. Hence, if $\mathfrak{I}$ is infinite, then the interval under consideration also has infinite width.

EXAMPLE 4.8. Let $\mathfrak{I}$ be a class of simple groups, let $p$ be an odd prime number and let $A=C_{p} \in \mathfrak{S} \backslash \mathfrak{I}$. Denote by $\mathfrak{L}$ the lower radical determined by $\mathfrak{I}$ and let $\mathfrak{U}=\mathcal{L}(\mathfrak{I} \cup A)$. If the class $\mathfrak{I}$ contains infinitely many groups $B$ with orders coprime to $p$ then one can check that the interval $[\mathfrak{L}, \mathfrak{U}]$ is of infinite height and width. This situation can happen in the following cases:

- $\quad \mathfrak{I}$ contains an infinite number of simple abelian groups;

- $\quad p=3$ and $\mathfrak{I}$ contains infinitely many Suzuki groups $S z(q)$ (see [6]);

- $\quad p>3$ and $\mathfrak{I}$ contains infinitely many groups $\operatorname{PSL}(2, q)$, where $q$ is not congruent to 0,1 or -1 modulo $p$ (see [6]).

The following proposition is a consequence of the above examples.

Proposition 4.9. Let $\mathfrak{I} \neq \subseteq$ be an infinite class of simple groups. Then, up to some exceptions, there exists $A \in \mathfrak{S} \backslash \mathfrak{I}$ such that the interval $[\mathcal{L} \mathfrak{I}, \mathcal{L}(\mathfrak{I} \cup A)]$ has infinite height and infinite width.

Proof. If there exists a nonabelian group $A \in \mathfrak{S} \backslash \mathfrak{I}$ then we are in the case of Example 4.7.

If every simple group outside of $\mathfrak{I}$ is abelian, and for some $C_{p}$ outside of $\mathfrak{I}$ there exist infinitely many groups in $\mathfrak{I}$ with orders not divisible by $p$, then we can apply Example 4.8.

Problem. Let $\mathfrak{I} \subseteq \mathfrak{S}$ be a proper subclass and $A \in \mathfrak{S} \backslash \mathfrak{I}$. Describe the intervals $\left[\mathcal{L} \mathfrak{I}, \mathcal{U}^{\prime} \mathfrak{I}\right]$ and $[\mathcal{L} \mathfrak{I}, \mathcal{L}(\mathfrak{I} \cup A)]$ as lattices. 
REMARK. Most of the above results can be repeated in the case of the subclass $\overline{\mathcal{E}} \mathfrak{I} \subset \mathfrak{F}=\overline{\mathcal{E}} \mathfrak{S}$, where $\mathfrak{I} \subseteq \subseteq$ is any subclass of simple groups. Many results on lattices of radicals from this paper can be formulated and proved in a categorical language, by adding to axioms in [16] some finiteness conditions, similar to the Jordan-Hölder theorem. These conditions should however be stronger than those from [17].

\section{References}

[1] C. Bagiński and A. Stocka, 'Finite groups with $L$-free lattices of subgroups', Illinois J. Math. 52 (2008), 887-900.

[2] K. Doerk and T. Hawkes, Finite Soluble Groups (Walter de Gruyter, Berlin-New York, 1992).

[3] B. J. Gardner, Radical Theory, Pitman Research Notes in Mathematics, 198 (Longman Sci. \& Tech, 1989).

[4] B. J. Gardner and R. Wiegandt, Radical Theory of Rings, Monographs and Textbooks in Pure and Applied Mathematics, 261 (Marcel Dekker, New York, 2004).

[5] G. Grätzer, General Lattice Theory, 2nd edn (Birkhäuser, Basel, 1998).

[6] B. Huppert and N. Blackburn, Finite Groups III (Springer, Berlin, 1982).

[7] J. Krempa and I. Malinowska, 'On Kurosh-Amitsur radicals of finite groups', An. Ştiinţ. Univ. 'Ovidius', Constanţa, Ser. Mat. 19(1) (2011), 175-190.

[8] J. Krempa and A. Sakowicz, 'On uniform dimension of finite groups', Colloq. Math. 89(2) (2001), 223-231.

[9] J. Krempa and B. Terlikowska-Osłowska, On Uniform Dimension of Lattices, Contributions to General Algebra, 9 (Teubner, Stuttgart, 1995), pp. 219-230.

[10] J. C. Lennox and S. E. Stonehewer, Subnormal Subgroups of Groups (Clarendon Press, Oxford, 1987).

[11] I. A. Malinowska, 'On finite nearly uniform groups', Publ. Math. Debrecen 69 (2006), 155-169.

[12] P. M. Neumann, 'On the structure of standard wreath product of groups', Math. Z. 84 (1964), 343-373.

[13] E. R. Puczyłowski, 'Some questions concerning radicals of associative rings', in: Theory of Radicals (Szekszard 1991) (North-Holland, Amsterdam, 1993), pp. 209-227.

[14] L. A. Shemetkov, A. N. Skiba and N. N. Vorob'ev, 'On lattices of formations of finite groups', Algebra Colloq. 17 (2010), 557-564.

[15] R. L. Snider, 'Lattices of radicals', Pacific J. Math. 40 (1972), 207-220.

[16] B. Terlikowska-Osłowska, 'Category with a selfdual set of axioms', Bull. Acad. Polon. Sci. 25 (1977), 1207-1214.

[17] A. Widiger, 'Lattices of radicals for hereditary artinian rings', Math. Nachr. 84 (1978), 301-309.

JAN KREMPA, Institute of Mathematics,

University of Warsaw ul. Banacha 2, 02-097 Warszawa, Poland

e-mail: jkrempa@mimuw.edu.pl

IZABELA AGATA MALINOWSKA, Institute of Mathematics, University of Białystok, ul. Akademicka 2, 15-267 Białystok, Poland e-mail: izabelam@math.uwb.edu.pl 